\title{
Instabilities in buoyant flows under localized heating
}

\author{
M. C. Navarro \\ Departamento de Matemáticas, Facultad de Ciencias Químicas, Universidad de Castilla-La Mancha, 13071 \\ Ciudad Real, Spain
}

\author{
A. M. Mancho ${ }^{\text {a) }}$ \\ Departamento de Matemáticas, IMAFF, Consejo Superior de Investigaciones Científicas, Serrano 121, \\ 28006 Madrid, Spain \\ H. Herrero \\ Departamento de Matemáticas, Facultad de Ciencias Químicas, Universidad de Castilla-La Mancha, 13071 \\ Ciudad Real, Spain
}

(Received 11 May 2006; accepted 9 February 2007; published online 18 April 2007)

\begin{abstract}
We study, from the numerical point of view, instabilities developed in a fluid layer with a free surface in a cylindrical container which is nonhomogeneously heated from below. In particular, we consider the case in which the applied heat is localized around the origin. An axisymmetric basic state appears as soon as a nonzero horizontal temperature gradient is imposed. The basic state may bifurcate to different solutions depending on vertical and lateral temperature gradients and on the shape of the heating function. We find different kinds of instabilities: extended patterns growing on the whole domain, which include those known as targets, and spiral waves. Spirals are present even for infinite Prandtl number. Localized structures both at the origin and at the outer part of the cylinder may appear either as Hopf or stationary bifurcations. An overview of the developed instabilities as functions of the dimensionless parameters is presented in this article. (C) 2007 American Institute of Physics. [DOI: 10.1063/1.2714295]
\end{abstract}

\begin{abstract}
Spiral and target wave instabilities in Rayleigh-Bérnard convection were experimentally found in the early 1990s and several theoretical mechanisms have been proposed to justify their appearance. In this article we study the instabilities generated in the flow by a nonuniform heating function. In this setup spiral and target waves are the linearly growing modes of nontrivial axisymmetric basic states. We find that localized structures also may appear. These patterns grow at different positions in the domain depending on the basic flow from which they bifurcate. This article reports a systematical numerical study of these transitions.
\end{abstract}

\section{INTRODUCTION}

Instabilities and pattern formation in buoyant flows have been extensively studied in recent years. Classically, heat is applied uniformly from below ${ }^{1}$ and the conductive solution (also called the basic solution) becomes unstable for a critical vertical temperature gradient beyond a certain threshold. Typically, instabilities in Rayleigh-Bénard convection lead to roll patterns, however spiral waves have also been reported, in both theoretical and experimental results. The first experimental observations on spirals ${ }^{2,3}$ in Rayleigh-Bénard convection were rather surprising as they were carried out for a set of external parameter values in which parallel rolls should be stable. In experiments, spirals have been reported for finite Prandtl number ${ }^{4}$ and both single-armed and multiarmed giant

\footnotetext{
a) Author to whom correspondence should be addressed.

Fax: +34 91 5854894. Electronic mail: a.m.mancho@mat.csic.es
}

spirals, and spiral chaos, have been observed. In Ref. 5 transitions between spiral and target states are reported. The first theoretical studies attempting to justify spiral patterns in Rayleigh-Bénard convection were performed only on model equations such as Swift-Hohenberg-type equations. ${ }^{6,7}$ These results were confirmed by later simulations of the full governing equations. 8,9

A setup for natural convection more general than that of uniform heating consists of a basic dynamic flow imposed by a nonzero horizontal temperature gradient which may be either constant or not. ${ }^{10-13,17,19,25-29,32-37}$ Numerical results obtained in the former setup in an annular domain show the importance of both vertical and horizontal temperature gradients in the development of instabilities. ${ }^{14-16,31,35}$ In this paper we consider a cylindrical domain with localized Gaussian-like heating at the bottom. Correspondingly, apart from vertical and horizontal temperature gradients, a heat parameter related to the shape of the heating profile at the bottom is introduced. Our results show that many different types of instabilities may appear depending on the basic flow and external parameters. We study how instabilities depend on dimensionless parameters and we recover many results such as those described for the classical uniform heating case. Giant, single-armed spiral waves appear and their instability threshold is close to those of patterns referred to as targets. Localized and extended spirals are identified and both of them are found for finite and infinite Prandtl number. Moreover, stationary patterns appear, which have local and extended structure. 


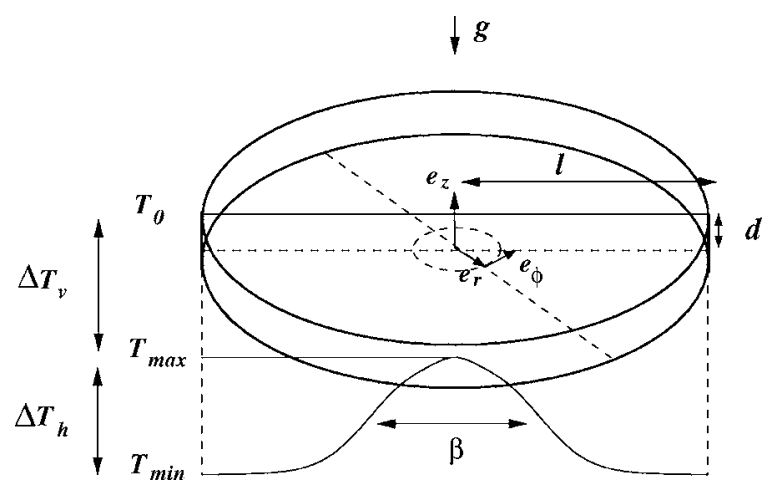

FIG. 1. Physical setup.

The paper is organized as follows. Section II describes the physical setup, the general mathematical formulation of the problem in a dimensionless form for basic solutions, and their linear stability analysis. Section III explains the numerical method in detail and presents a test of convergence. In Sec. IV the numerical results on both the basic and growing perturbations are discussed and conclusions are presented.

\section{FORMULATION OF THE PROBLEM}

The physical setup (see Fig. 1) consists of a horizontal fluid layer in a cylindrical container of radius $l$ ( $r$ coordinate) and depth $d$ ( $z$ coordinate). The upper surface is open to the atmosphere and the bottom plate is rigid. At $z=0$ the imposed temperature has a Gaussian profile which takes the value $T_{\max }$ at $r=0$ and the value $T_{\min }$ at the outer part $(r=l)$. The environmental temperature is $T_{0}$. We define $\Delta T_{v}=T_{\max }$ $-T_{0}, \Delta T_{h}=T_{\max }-T_{\min }$, and $\delta=\Delta T_{h} / \Delta T_{v}$.

In the equations governing the system, $u_{r}, u_{\phi}$, and $u_{z}$ are the components of the velocity field $\mathbf{u}, T$ is the temperature, $p$ is the pressure, $r$ is the radial coordinate, and $t$ is the time. The magnitudes are expressed in dimensionless form after rescaling in the following form: $\mathbf{r}^{\prime}=\mathbf{r} / d, t^{\prime}=\kappa t / d^{2}, \mathbf{u}^{\prime}$ $=d \mathbf{u} / \kappa, p^{\prime}=d^{2} p /\left(\rho_{0} \kappa \nu\right), \Theta=\left(T-T_{0}\right) / \Delta T_{v}$. Here $\mathbf{r}$ is the position vector, $\kappa$ the thermal diffusivity, $\nu$ the kinematic viscosity of the liquid, and $\rho_{0}$ is the mean density at the environment temperature $T_{0}$. After rescaling the domain $\Omega_{1}$ $=[0, l] \times[0, d]$ is transformed into $\Omega_{2}=[0, \Gamma] \times[0,1]$, where $\Gamma=l / d$ is the aspect ratio.

The system evolves according to the momentum and the mass balance equations, and to the energy conservation principle, which in dimensionless form are (the primes in the corresponding fields have been dropped)

$$
\begin{aligned}
& \nabla \cdot \mathbf{u}=0, \\
& \partial_{t} \Theta+\mathbf{u} \cdot \nabla \Theta=\nabla^{2} \Theta, \\
& \partial_{t} \mathbf{u}+(\mathbf{u} \cdot \nabla) \mathbf{u}=\operatorname{Pr}\left(-\nabla p+\nabla^{2} \mathbf{u}+\mathrm{R} \Theta \mathbf{e}_{\mathbf{z}}\right),
\end{aligned}
$$

where the operators and fields are expressed in cylindrical coordinates and the Oberbeck-Bousinesq approximation has been used. Here $\mathbf{e}_{\mathbf{z}}$ is the unit vector in the $z$ direction. The following dimensionless numbers have been introduced: the Prandtl number $\operatorname{Pr}=\nu / \kappa$ and the Rayleigh number $\mathrm{R}$ $=g \alpha \Delta T_{v} d^{3} / \kappa \nu$, which represents the buoyant effect. In these expressions $\alpha$ is the thermal expansion coefficient and $g$ is the gravity constant.

We discuss now the boundary conditions (bc). The top surface is flat, which implies the following condition on the velocity:

$$
u_{z}=0 \quad \text { on } z=1,
$$

and free slip,

$$
\partial_{z} u_{r}=0, \quad \partial_{z} u_{\phi}=0 \quad \text { on } z=1 .
$$

The lateral and bottom walls are rigid, so

$$
\begin{array}{ll}
u_{r}=u_{\phi}=u_{z}=0 & \text { on } z=0, \\
u_{r}=u_{\phi}=u_{z}=0 & \text { on } r=\Gamma .
\end{array}
$$

For temperature we consider the dimensionless form of Newton's law for heat exchange at the surface,

$$
\partial_{z} \Theta=-\mathrm{B} \Theta \quad \text { on } z=1,
$$

where B is the Biot number. The lateral wall is insulating,

$$
\partial_{r} \Theta=0 \quad \text { on } r=\Gamma .
$$

At the bottom a Gaussian profile is imposed,

$$
\begin{aligned}
\Theta= & 1-\delta\left(e^{(1 / \beta)^{2}}-e^{\left(1 / \beta-(r / \Gamma)^{2} 1 / \beta\right)^{2}}\right) /\left(e^{(1 / \beta)^{2}}-1\right) \\
& \text { on } z=0 .
\end{aligned}
$$

This choice of this equation is justified as follows. At $r=0$ the rescaling makes $\Theta=1$ (see Fig. 1) and at $r=\Gamma$ Eq. (10) imposes $\Theta=1-\delta$. Therefore, if $\delta>0$, the center of the cell $(r=0)$ at the bottom is hotter than the outer part $(r=\Gamma)$, while the opposite occurs for $\delta<0$. If $\delta=0$ the classical uniform heating case is recovered. This is represented in Fig. 2(b) for different positive and negative $\delta$ values. The parameter $\beta$ is a measure of the width of the profile. Small $\beta$ values correspond to sharp profiles very localized at the origin while large $\beta$ correspond to widespread inhomogeneities along the bottom boundary. Figure 2(a) displays several plots with the parameter $\beta$ varying. In the limit $\beta \rightarrow \infty$, Eq. (10) becomes $\Theta=1-\delta\left(2(r / \Gamma)^{2}-(r / \Gamma)^{4}\right)$. The convergence to this limit for $\beta \rightarrow \infty$ ensures the continuity of the derivatives of the temperature at $r=\Gamma$ and $z=0$, which is not satisfied by a purely Gaussian function. In practice this limit is reached with $\beta$ $=5$, which is the maximum value we adopt in our study. Very sharp profiles at very small $\beta$ values require large expansions and are left for future studies. We only study moderately localized heating with $\beta>0.5$.

The use of cylindrical coordinates, which are singular at $r=0$, requires regularity conditions on velocity, pressure, and temperature fields. In general, these conditions are expressed as follows: ${ }^{18}$

$$
\frac{\partial\left(u_{r} e_{r}+u_{\phi} e_{\phi}\right)}{\partial \phi}=\partial_{\phi} u_{z}=\partial_{\phi} p=\partial_{\phi} \Theta=0 \quad \text { on } r=0,
$$

where $e_{r}$ and $e_{\phi}$ are the unit vectors in the $r$ and $\phi$ directions, respectively. To summarize, the dimensionless equations contain these external parameters $(\mathrm{R}, \Gamma, \operatorname{Pr}, \delta, \mathrm{B}, \beta)$. Our aim is to describe the types of bifurcations that appear when some of these dimensionless quantities are varied. 

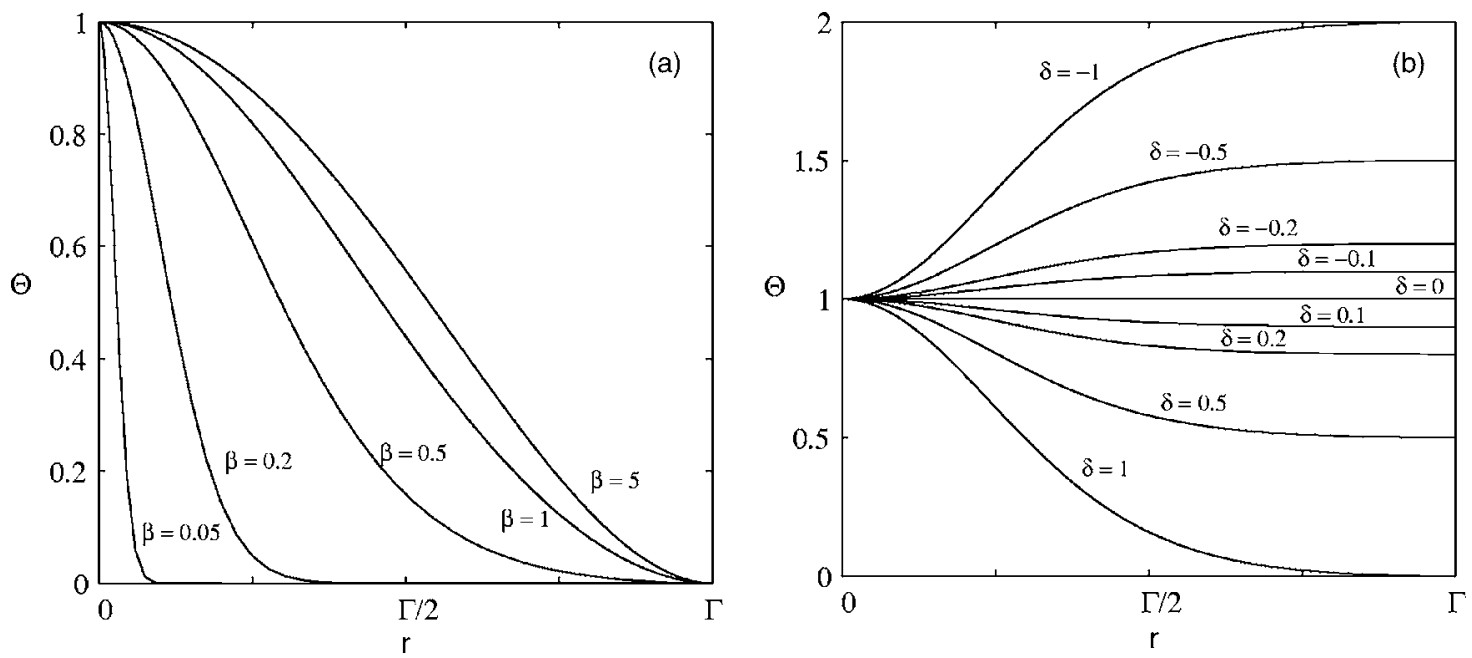

FIG. 2. Temperature boundary conditions at the bottom for $\Gamma=10$, for (a) $\delta=1$ and different values of $\beta$; (b) $\beta=0.5$ and different values of $\delta$.

\section{A. Basic state and linear stability analysis}

The horizontal temperature gradient at the bottom (i.e, $\delta \neq 0$ ) settles into a stationary convective motion, which is called "basic state." It is a time-independent solution to the stationary problem obtained from Eqs. (1)-(3). The basic state is axisymmetric and therefore depends only on $r-z$ coordinates (i.e., all $\phi$ derivatives are zero). The velocity field of the basic flow is restricted to $\mathbf{u}=\left(u_{r}, u_{\phi}=0, u_{z}\right)$. The regularity conditions (11) now become

$$
u_{r}=\partial_{r} u_{z}=\partial_{r} p=\partial_{r} \Theta=0 \quad \text { on } r=0 .
$$

We have solved numerically the simplified equations for the basic state together with its boundary conditions. We use a Chebyshev collocation method, the details of which are given in Sec. III.

The stability of the basic state is studied by perturbing it with a vector field depending on the $r, \phi$, and $z$ coordinates in a fully three-dimensional (3D) analysis,

$$
\begin{aligned}
& u_{r}(r, \phi, z)=u_{r}^{b}(r, z)+\bar{u}_{r}(r, z) \exp (i k \phi+\lambda t), \\
& u_{\phi}(r, \phi, z)=u_{\phi}^{b}(r, z)+\bar{u}_{\phi}(r, z) \exp (i k \phi+\lambda t), \\
& u_{z}(r, \phi, z)=u_{z}^{b}(r, z)+\bar{u}_{z}(r, z) \exp (i k \phi+\lambda t), \\
& \Theta(r, \phi, z)=\Theta^{b}(r, z)+\bar{\Theta}(r, z) \exp (i k \phi+\lambda t), \\
& p(r, \phi, z)=p^{b}(r, z)+\bar{p}(r, z) \exp (i k \phi+\lambda t) .
\end{aligned}
$$

Here the superscript $b$ indicates the corresponding quantity in the basic state and the bar refers to the perturbation. We have considered Fourier mode expansions in the angular direction, because along it boundary conditions are periodic. Expressions (13)-(17) are substituted into basic equations (1)-(3) and the resulting system is linearized. Boundary conditions for perturbations $\left(\bar{u}_{r}, \bar{u}_{\phi}, \bar{u}_{z}, \bar{\Theta}, \bar{p}\right)$ are found by substituting (13)-(17) into (4)-(11). The regularity conditions (11) depend now on the wave number $k$,

$$
\begin{aligned}
& \bar{u}_{r}=\bar{u}_{\phi}=\frac{\partial \bar{u}_{z}}{\partial r}=\frac{\partial \bar{\Theta}}{\partial r}=\frac{\partial \bar{p}}{\partial r}=0, \quad \text { for } k=0, \\
& \bar{u}_{r}+i \bar{u}_{\phi}=\bar{u}_{z}=\bar{\Theta}=\bar{p}=0, \quad \text { for } k=1, \\
& \bar{u}_{r}=\bar{u}_{\phi}=\bar{u}_{z}=\bar{\Theta}=\bar{p}=0, \quad \text { for } k \neq 0,1 .
\end{aligned}
$$

The resulting problem is an eigenvalue problem in $\lambda$. If $\operatorname{Re}(\lambda)<0$ for all eigenvalues the basic state is stable; while if there exists a value of $\lambda$ for which $\operatorname{Re}(\lambda)>0$ the basic state becomes unstable. The condition $\operatorname{Re}(\lambda)=0$ may be satisfied for certain values of the external parameters $(\mathrm{R}, \Gamma, \operatorname{Pr}, \delta, \mathrm{B}, \beta)$, which define the critical threshold. At the critical threshold, a stationary bifurcation takes place if $\operatorname{Im}(\lambda)=0$, while it is a Hopf bifurcation if $\operatorname{Im}(\lambda) \neq 0$.

\section{NUMERICAL METHOD}

We have solved both the basic state and linear stability problems as stated in (1)-(3), i.e., in primitive variables formulation by expanding the fields with Chebyshev polynomials (see Ref. 20). Using this technique, the problem of spurious modes for pressure arises, ${ }^{20,21}$ which we have solved using the method proposed in Ref. 18, which requires additional boundary conditions. They are obtained from the continuity equation at $z=0$ and the normal component of the momentum equations on $r=\Gamma$ and $z=1$,

$$
\begin{aligned}
& \nabla \cdot \mathbf{u}=0, \quad \text { on } z=0, \\
& \operatorname{Pr}^{-1}\left(\frac{\partial u_{r}}{\partial t}+u_{r} \frac{\partial u_{r}}{\partial r}+\frac{u_{\phi}}{r} \frac{\partial u_{r}}{\partial \phi}+u_{z} \frac{\partial u_{r}}{\partial z}-\frac{u_{\phi}^{2}}{r}\right) \\
& =-\frac{\partial p}{\partial r}+\Delta u_{r}-\frac{u_{r}}{r^{2}}-\frac{2}{r^{2}} \frac{\partial u_{\phi}}{\partial \phi} \quad \text { on } r=\Gamma,
\end{aligned}
$$




$$
\begin{array}{r}
\operatorname{Pr}^{-1}\left(\frac{\partial u_{z}}{\partial t}+u_{r} \frac{\partial u_{z}}{\partial r}+\frac{u_{\phi}}{r} \frac{\partial u_{z}}{\partial \phi}+u_{z} \frac{\partial u_{z}}{\partial z}\right) \\
=-\frac{\partial p}{\partial z}+\Delta u_{z}+\operatorname{R} \Theta \quad \text { on } z=1
\end{array}
$$

where $\Delta=r^{-1} \partial / \partial r(r \partial / \partial r)+r^{-2} \partial^{2} / \partial \phi^{2}+\partial^{2} / \partial z^{2}$.

\section{A. Basic state}

We have solved numerically the stationary axisymmetric version of Eqs. (1)-(3) together with the boundary conditions, by treating the nonlinearity with a Newton-like iterative method. In a first step the nonlinearity was neglected and a solution was found by solving the linear system: $u_{r}^{0}, u_{z}^{0}, p^{0}$, $\Theta^{0}$. This solution was corrected by perturbation fields: $u_{r}^{1}$ $=u_{r}^{0}+\bar{u}_{r}, u_{z}^{1}=u_{z}^{0}+\bar{u}_{z}, p^{1}=p^{0}+\bar{p}$, and $\Theta^{1}=\Theta^{0}+\bar{\Theta}$. These expressions are introduced into the equations, which are linearized around the approach at step 0. This procedure is repeated with the new solution and so on. The convergence criterion considered to stop the iterative procedure is that the $l^{2}$ norm of the computed perturbation should be less than $10^{-9}$.

At each step the resulting linear system for perturbations is solved by expanding any unknown perturbation field $x$ in Chebyshev polynomials,

$$
x=\sum_{l=0}^{L-1} \sum_{n=0}^{N-1} a_{l n}^{x} T_{l}(r) T_{n}(z) .
$$

Chebyshev polynomials are defined in the interval $[-1,1]$ and therefore, for computational convenience, the domain $\Omega_{2}=[0, \Gamma] \times[0,1]$ is transformed into $\Omega=[-1,1] \times[-1,1]$. This is just a linear transformation where the origin $r=0, z$ $=0$ is translated to the position $r=-1, z=-1$ and the upper right corner of the cell $r=\Gamma, z=1$ to $r=1, z=1$. From now on boundaries will be referred to in this new reference system, so $r$ and $z$ will also take negative values. This change of coordinates introduces scaling factors in equations and boundary conditions, which are not explicitly given here. There are $4 \times L \times N$ unknowns, which are determined by a collocation method. In particular, expansions (24) are substituted into the linearized, stationary, axisymmetric equations and boundary conditions, and these are posed at the GaussLobatto collocation points $\left(r_{j}, z_{i}\right)$,

$$
\begin{aligned}
& r_{j}=\cos \left(\left(\frac{j-1}{L-1}-1\right) \pi\right), \quad \forall j=1, \ldots, L, \\
& z_{i}=\cos \left(\left(\frac{i-1}{N-1}-1\right) \pi\right), \quad \forall i=1, \ldots, N .
\end{aligned}
$$

Evaluation rules are as follows: The conveniently simplified Eqs. (1)-(3) are evaluated at nodes $i=2, \ldots, N-1, j$ $=2, \ldots, L-1$. This provides $4 \times N \times L-8(L-2)-8 N$ equations; the boundary conditions at $z=-1,(6)$ and (10) are evaluated at $i=1, j=2, \ldots, L-1$; the boundary conditions at $z=1,(4),(5)$, and (8) at $i=N, j=2, \ldots, L-1$; the boundaries at $r=-1,(12)$ at $i=1, \ldots, N, j=1$; finally, the boundaries at $r=1,(7)$ and (9) at $i=1, \ldots, N, j=L$. These conditions provide an additional $6(L-2)+7 N$ equations. The system is completed with additional boundary conditions that eliminate spurious modes for pressure as is proven in Refs. 18 and 22. In particular, additional $(L-2)+(L-1)+(N-1)$ conditions are obtained if Eq. (21) at $z=-1$ is evaluated at nodes $i=1, j=2, \ldots, L-1$; Eq. (23) at $z=1$ is evaluated at nodes $i$ $=N, j=2, \ldots, L$; Eq. (22) at $r=1$ is evaluated at $i=1, \ldots, N$ $-1, j=L$. With these rules the matrix of order $P \times P$ with $P=4 \times L \times N$ associated with the linear algebraic system is singular, due to the fact that pressure with the imposed conditions is defined up to an additive constant. ${ }^{18,22}$ To fix this constant the boundary condition (22) at node $i=N-2, j=L$ is replaced by a Dirichlet condition for pressure (i.e, $p=0$ at $i$ $=N-2, j=L)$. In this way, at each iteration a linear system of the form $A \mathbf{X}=B$ is obtained, where $\mathbf{X}$ is a vector containing $4 \times L \times N$ unknowns and $A$ is a full rank matrix of order $P$ $\times P$. This can be easily solved with standard routines.

\section{B. Linear stability analysis}

The eigenvalue problem described in Sec. III A is discretized by expanding perturbations (13)-(17) in a truncated series of orthonormal Chebyshev polynomials as we did with the basic state. These expressions are substituted into the linearized version of Eqs. (1)-(3) and the boundary and regularity conditions (4)-(11). We use as in the previous section a collocation method where equations are evaluated at the Gauss-Lobatto points. Evaluation rules are analogous except that, now, the regularity conditions are replaced by those expressed in (18)-(20). We notice that for $k=1$ there are only four boundary conditions at $r=-1$, and therefore, in order to fit the unknowns with the equations we have reduced the expansion range of the $u_{\phi}$ field by one order,

$$
u_{\phi}=\sum_{l=0}^{L-2} \sum_{n=0}^{N-1} a_{l n}^{v} T_{l}(r) T_{n}(z) .
$$

In this way for $k=1$ we have $P=4 \times N \times L+N \times(L-1)$ unknowns and equations while there are $P=5 \times L \times N$ for $k$ $>1$ and $P=4 \times L \times N$ for $k=0$. For the eigenvalue problem these rules are explicitly detailed in Ref. 18, with the only difference being that here the equations are linearized around a nontrivial basic state instead of around a conductive solution.

The eigenvalue problem is then transformed into its discrete form,

$$
A \mathbf{w}=\lambda B \mathbf{w},
$$

where $\mathbf{w}$ is a vector that contains $P$ unknowns, and $A$ and $B$ are $P \times P$ matrices. The discrete eigenvalue problem (27) has a finite number of eigenvalues $\lambda_{i}$. The stability condition explained in the previous section must be imposed now upon $\lambda_{\max }$, where $\lambda_{\max }=\max \operatorname{Re}\left(\lambda_{i}\right)$. In Ref. 23 details on how to solve this problem efficiently are given.

As in Ref. 14, we have carried out a test on the convergence of the method that lets us be sure of the correctness of the results. Table I shows some results on convergence rates. We find that expansions of order $33 \times 9$ are enough to ensure accuracy within $1 \%$. 
TABLE I. Critical Rayleigh numbers $\mathbf{R}_{c}$ for different orders of expansions in Chebyshev polynomials. The critical wave number is $k_{c}=0$ and the parameters are $\Gamma=10, \operatorname{Pr}=0.4, \delta=0.05, \mathrm{~B}=0.05$, and $\beta=5$.

\begin{tabular}{llllll}
\hline \hline & $N=9$ & $N=11$ & $N=13$ & $N=15$ & $N=17$ \\
\hline$L=19$ & 19069 & 19070 & 19070 & 19070 & 19070 \\
$L=25$ & 19055 & 19056 & 19056 & 19056 & 19056 \\
$L=29$ & 19065 & 19066 & 19066 & 19066 & 19066 \\
$L=33$ & 19063 & 19064 & 19064 & 19064 & 19064 \\
$L=37$ & 19063 & 19064 & 19064 & 19064 & 19064 \\
\hline
\end{tabular}

\section{NUMERICAL RESULTS}

In this section we describe the numerical results found for different values of the external parameters. The problem formulated through Eqs. (1)-(11) depends on the dimensionless numbers $(\mathrm{R}, \Gamma, \operatorname{Pr}, \delta, \mathrm{B}, \beta)$. In particular we are interested in analyzing how those parameters related to heat conditions impose basic flows leading to a great variety of instabilities. Some of them such as giant spirals or targets have been described already in experiments for the case of Rayleigh-Bénard convection with uniform heating. ${ }^{2,45}$
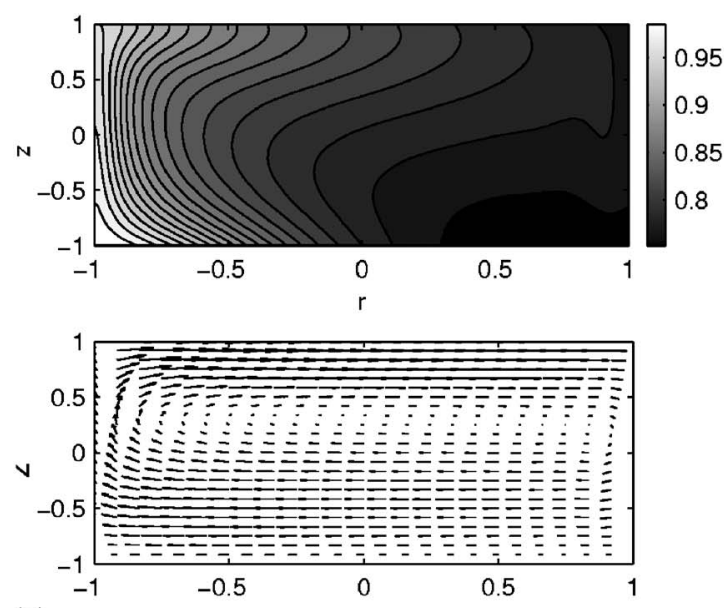

(a)
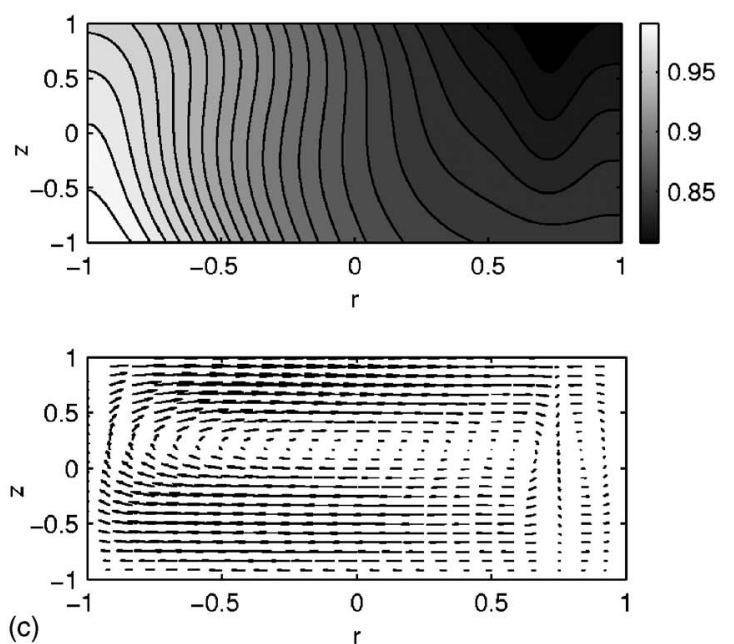

Issues related to horizontal and vertical temperature differences have already been addressed in Refs. 14-16, but here we study for the first time the influence of the shape parameter $\beta$, which measures the width of the heating. We restrict ourselves to large and medium $\beta$ values, i.e., $\beta$ $>0.5$. In this region, which corresponds to moderately localized and wide heating, the numerical method has good convergence and among other instabilities a giant spiral is observed. Smaller $\beta$ (very sharp profiles) values will be addressed in future works. We also analyze the influence of the parameter $\delta$, which measures the quotient between lateral and vertical temperature gradients. Figure 2(a) illustrates the dependence of the temperature boundary condition on $\beta$, while Fig. 2(b) shows its dependence on $\delta$.

\section{A. Basic states}

We have solved numerically the stationary axisymmetric version of Eqs. (1)-(3) together with the boundary conditions, as explained in Sec. III A. Different basic state solutions are obtained depending on the parameters. Figure 3(a) displays a return flow with clearly inverted temperature gradients and a single roll on the velocity field. Figure 3(b)
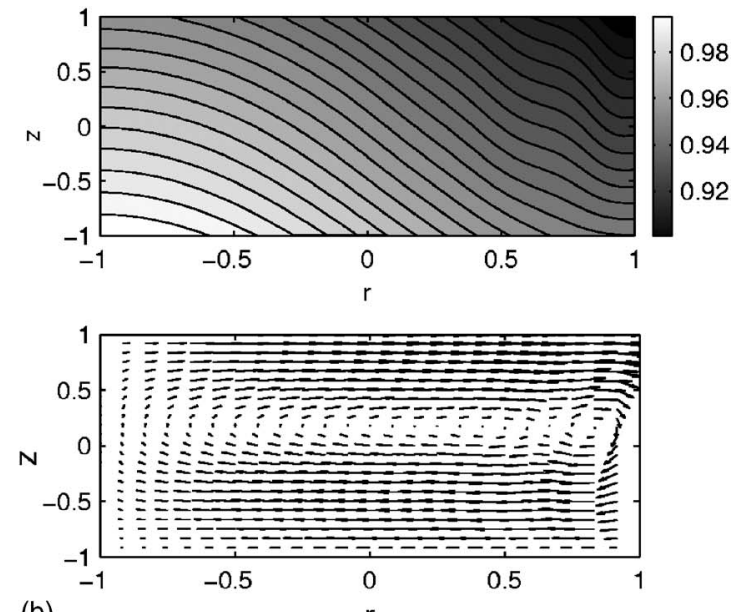

(b)
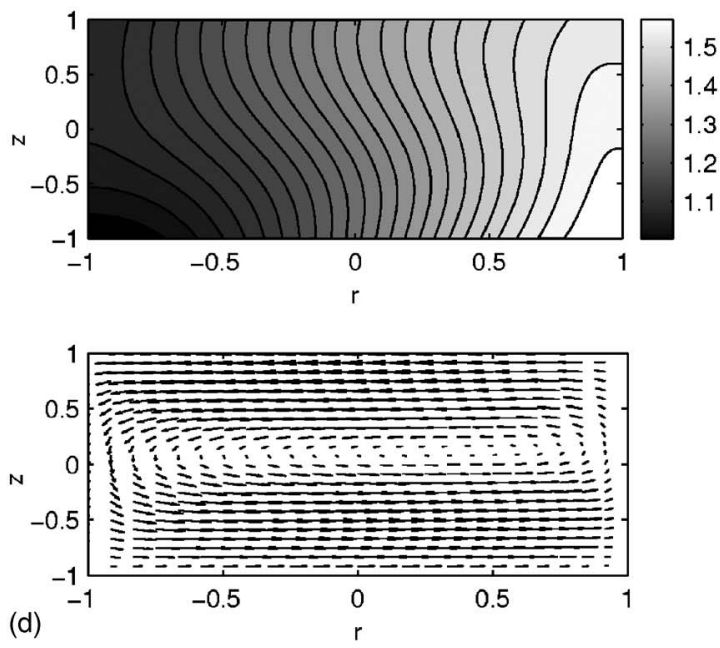

FIG. 3. Isotherms and velocity field of the basic state corresponding to values of the parameters $(\mathrm{a}) \mathrm{R}=356880, \Gamma=10, \operatorname{Pr}=0.4, \mathrm{~B}=0.05, \delta=0.25$, and $\beta$ $=0.5$; (b) $\mathrm{R}=19064, \Gamma=10, \mathrm{Pr}=0.4, \mathrm{~B}=0.05, \delta=0.05$, and $\beta=5$; (c) $\mathrm{R}=30377, \Gamma=10, \operatorname{Pr}=0.4, \mathrm{~B}=0.05, \delta=0.15$, and $\beta=0.5$; $(\mathrm{d}) \mathrm{R}=10250, \Gamma=10, \operatorname{Pr}$ $=0.4, \mathrm{~B}=0.05, \delta=-0.6$, and $\beta=5$. 


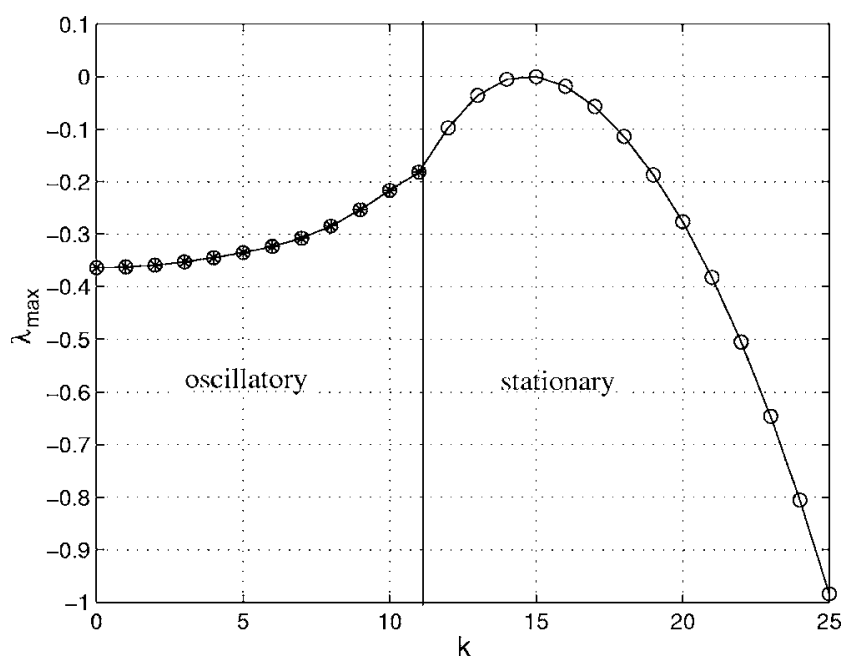

FIG. 4. Maximum real part of the growth rate $\lambda$ as a function of $k$ for basic state at threshold $\mathrm{R}_{c}=15095$; the rest of the parameters are $\Gamma=10, \operatorname{Pr}=0.4$, $\mathrm{B}=0.05, \delta=0.05$, and $\beta=0.5$. The maximum determines the critical $k_{c}=15$. Void circles correspond to real eigenvalues while crossed ones stand for complex eigenvalues.

shows a linear temperature field with no inversions on the temperature gradient and several corotative rolls. Figures 3(c) and 3(d) display intermediate states. In Fig. 3(c) we see rather vertical temperature gradients with slight inversions and a slight corotative roll. Figure 3(d) displays a return flow where the outer part of the cylinder is hotter than the inner one. These basic flows become unstable for different spatial structures as we explain next.

\section{B. Instabilities}

We have studied numerically the linear stability of the numerical basic states following the explanations of Sec. III B. We consider the external parameter $\mathrm{R}$ as the control parameter. By control parameter we mean that instabilities are obtained when this parameter is varied while all the others are fixed. This occurs when $\lambda_{\max }(\mathrm{R})$ changes from a negative value to a positive one as $\mathrm{R}$ varies. The critical value is the value of $R_{c}$ for which $\lambda_{\max }\left(R_{c}\right)=0$. Figure 4 displays $\lambda_{\max }\left(\mathrm{R}_{c}\right)$ as a function of the wave number $k$ with all other parameters fixed. The eigenvalue with maximum real part corresponds to $k=15$, and as it is purely real, the bifurcation is stationary. Depending on the different parameters a great variety of instabilities are obtained, which are either stationary or oscillatory, with differently growing modes. These are analyzed next.

\section{Influence of $\beta$ and $\delta$}

In this section we study how the instabilities are affected by parameters related to the shape of the heating boundary condition at the bottom. The factor $\beta$ is related to the width of the Gaussian profile and $\delta$ is the quotient of horizontal and vertical temperature differences.

To start with we fix parameters $\Gamma=10, \operatorname{Pr}=0.4$, and $\mathrm{B}$ $=0.05$ and take $\mathrm{R}$ as the control parameter. With the previous parameter choice, and $\delta=0$, the classical Rayleigh-Bénard problem is recovered, for which the instability threshold is
$\mathrm{R}_{c}=14263.42$. The problem is proven to satisfy the principle of exchange of stabilities in Refs. 24 and 30 and it presents only real eigenvalues of which the most unstable has wave number $k_{c}=3$. If $\delta \neq 0$ a basic flow appears that leads to a problem which no longer presents only real eigenvalues. Figures 5(a) and 5(b) display critical values of $\mathrm{R}_{c}$ as a function of $\delta$ for $\beta=0.5$ (sharp localized heating) and $\beta=5$ (spread out heating), respectively. These functions are obtained for a discrete set of $\delta$ values in which $\delta=0$ is excluded and the evolution of the asymptotic limit $\delta \rightarrow 0$ has not been studied in detail. Critical wave numbers $k_{c}$ and the corresponding growing modes are shown in these figures, where void circles correspond to stationary bifurcations and crossed ones stand for oscillatory instabilities. Both figures show regions with qualitatively different behaviors, which are separated by vertical lines. In Fig. 5(a) for $\delta>0.15$ and in Fig. 5(b) for $\delta>0.055$ the critical $\mathrm{R}_{c}$ increases when $\delta$ decreases. At the $\delta$ limit values, the $\mathrm{R}$ threshold tends to grow so much that we have not found it. This suggests that there is an asymptote where the critical $\mathrm{R}_{c}$ tends to infinity. As $\delta$ is increased, the control parameter $\mathrm{R}$ decreases and so does the critical wave number. Comparing Figs. 5(a) and 5(b), which differ in their $\beta$ values, we notice more localized bifurcating structures for small values of $\beta$ (localized heating). This part of the diagram is called zone I. At different $\beta$ the critical $\mathrm{R}_{c}$ tends to infinity at different $\delta$ values. In Fig. 6 , the curve $C_{1}$ (solid line) that limits zone I marks the position of this asymptote in the $\beta-\delta$ plane. An example of a basic state in zone I is presented in Fig. 3(a). It corresponds to a return flow.

In Fig. 5(b), at $-0.05<\delta<-0.01$ and $0.01<\delta<0.055$, an oscillatory bifurcation is obtained with wave number $k$ $=0$. Simulations within the interval $-0.01<\delta<0.01$ have not been performed, except for the homogeneous case $\delta=0$, which would correspond to a different zone and which we indicate only by drawing a vertical line at $\delta=0$. This is detailed in Fig. 5(c), in which a zoom of this region is displayed. The critical $\mathrm{R}_{c}$ tends to infinity as $\delta$ tends to the outer interval limits. The oscillatory mode $k=1$ is just below the instability threshold but very close to it, at the same parameter values. Figure 7 displays the values of $\lambda_{\max }\left(R_{c}\right)$ as a function of the wave number $k$. The zero mode is the preferred one, but as the mode one is so close to it, it could be possible that both modes appear after the bifurcation. The mode $k=0$ corresponds to a target pattern [see Fig. 8(a)] and the mode $k=1$ to a giant one-armed spiral [see Fig. 8(b)]. These plots represent contours of the growing temperature field eigenfunction and their structures are quite similar to those described in Refs. 2-5. The zone where we find these behaviors is called zone II (see Fig. 6). We do not find such instabilities for $\beta=0.5$. In fact, they exist only for large enough $\beta$ values in a small interval around $\delta=0$ (excluding $\delta=0$ and maybe a smaller interval around it). This is clearly depicted in Fig. 6 in which the curve $C_{2}$ (dot-dashed line) limits zone II. Figure 3(b) displays an example of a basic state in zone II. It corresponds to a linear flow. In order to explore the linkage of these instabilities with experimental results such as those of Refs. 2-5, we relate the dimensionless parameters in Fig. 3(b) with a physical setup. We consider a cylindrical container with diameter $D=2 \mathrm{~cm}$ and 

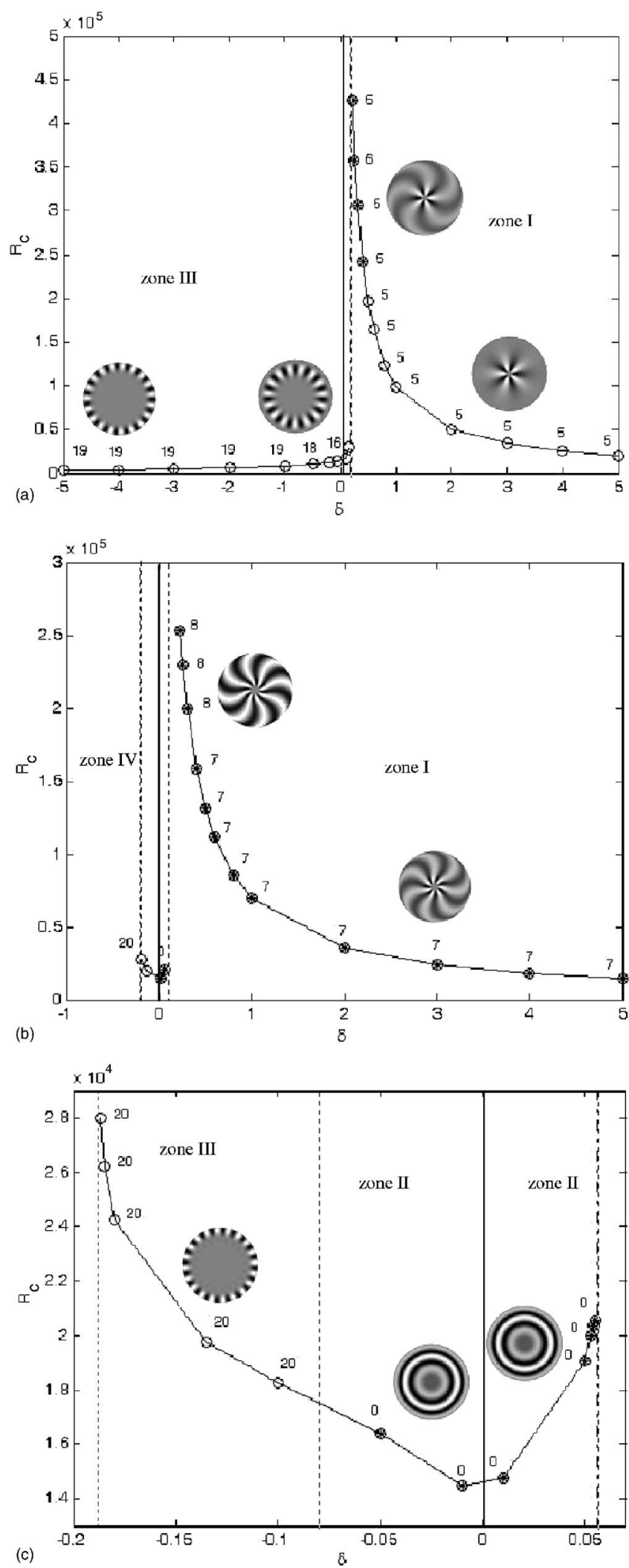

(c)

FIG. 5. (a) Critical R, wave number $k$ values and growing modes as a function of $\delta$ for $\beta=0.5$. The remaining external parameters are $\Gamma=10$, Pr $=0.4$, and $\mathrm{B}=0.05$; (b) the same for $\beta=5$. Void circles correspond to stationary instabilities while crossed ones stand for oscillatory instabilities. (c) A zoom of zones II and III of (b). The dashed-vertical lines correspond to the asymptotes and the thick vertical line at $\delta=0$ marks the uniform heating case.

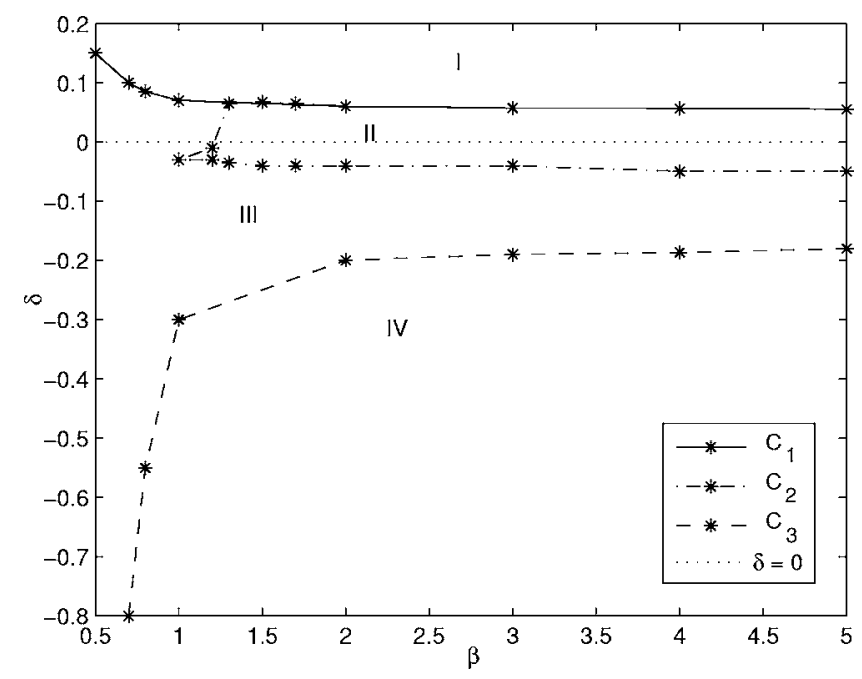

FIG. 6. Different zones of bifurcations in the $\beta-\delta$ plane. The rest of the parameters are $\Gamma=10, \operatorname{Pr}=0.4$, and $\mathrm{B}=0.05$. The dotted line at $\delta=0$ marks the uniform heating case.

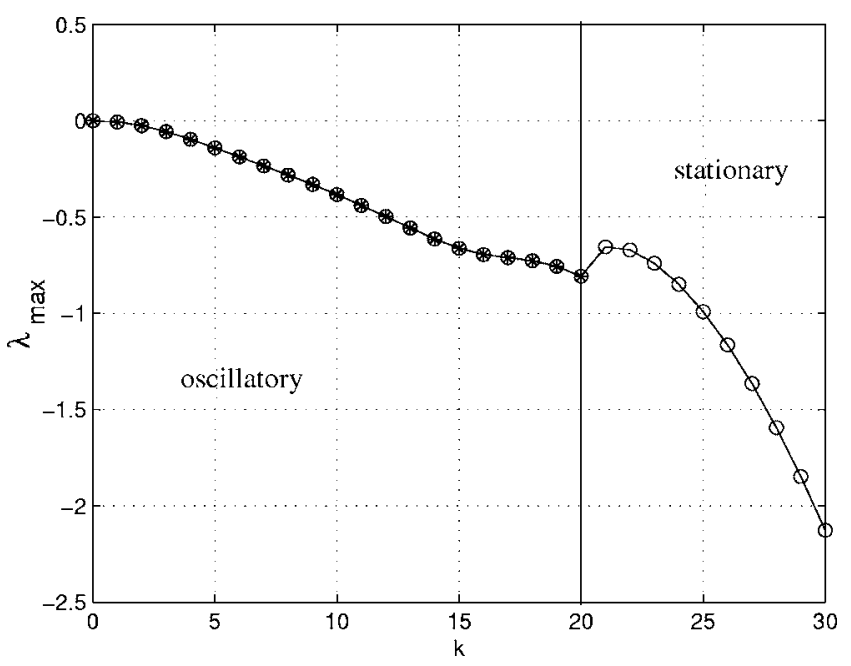

FIG. 7. Maximum real part of the growth rate $\lambda$ as a function of $k$ for basic state at threshold $\mathrm{R}_{c}=19064$ corresponding to the target $\left(k_{c}=0\right)$ and the giant one-armed spiral $(k=1)$ just below the instability threshold but very close to it. The rest of the parameters are $\Gamma=10, \operatorname{Pr}=0.04, B=0.05, \delta$ $=0.05$, and $\beta=5$. Void circles correspond to real eigenvalues while crossed ones stand for complex eigenvalues.

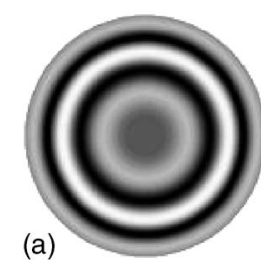

FIG. 8. (a) Target at the instability threshold $\mathrm{R}=19064$. The rest of the parameters are $\Gamma=10, \operatorname{Pr}=0.4, \mathrm{~B}=0.05, \delta=0.05$, and $\beta=5$. (b) Giant onearmed spiral just below the instability threshold but very close to it, at the same parameter values. (c) Giant one-armed spiral for $\operatorname{Pr}=\infty$. The rest of the parameters are $\mathrm{R}=4674, \Gamma=10, \mathrm{~B}=0.2, \delta=0.1$, and $\beta=5$. 
depth $d=1 \mathrm{~mm}$ (i.e., $\Gamma=10$ ). It contains a liquid with thermal diffusivity $\kappa=0.7 \times 10^{-7} \mathrm{~m}^{2} / \mathrm{s}$, kinematic viscosity $\nu=0.28$ $\times 10^{-7} \mathrm{~m}^{2} / \mathrm{s}$, and thermal expansion coefficient $\alpha=9.5223$ $\times 10^{-4}{ }^{\circ} \mathrm{C}^{-1}$ (i.e., $\mathrm{Pr}=0.4$ ). The gravity constant is $g$ $=9.81 \mathrm{~m} / \mathrm{s}^{2}$ and the heat exchange at the surface is $\mathrm{B}=0.05$ (this is the value assumed in our numerics but it is a rather difficult parameter to quantify in experiments). At the bottom a temperature profile with width $\beta=5$ and a small lateral temperature difference $\Delta T_{h}=0.2{ }^{\circ} \mathrm{C}$ is imposed. The instability threshold for target waves is obtained when the vertical temperature difference is $\Delta T_{v}=4{ }^{\circ} \mathrm{C}$ (i.e, $\mathrm{R}=19064, \delta$ $=0.05$ ). The lateral inhomogeneity is rather small: $0.2{ }^{\circ} \mathrm{C}$, compared with the vertical temperature gradient: $4{ }^{\circ} \mathrm{C}$, however it is enough to modify the type of instability which is different from that of the homogeneous heating case. The main difference between both cases is given by the basic flow depicted in Fig. 3(b) in which the maximum radial velocity is $0.021 \mathrm{~cm} / \mathrm{s}$. This is smaller but still comparable to those radial velocities shown in Figs. 3(a) or 3(c), which, with the same $\kappa$ and $d$, would be, respectively, 0.695 and $0.078 \mathrm{~cm} / \mathrm{s}$.

In Fig. 5(a) for $\delta<0.15$ and in Fig. 5(b) for -0.186 $<\delta<-0.05$, the bifurcations are stationary with a large wave number that increases when $\delta$ decreases. The bifurcating structures are localized at the outer part of the cylinder as Figs. 5(a) and 5(b) display. In this range we can observe how for $\beta=0.5$ the critical $\mathrm{R}_{c}$ decreases with $\delta$ while for $\beta=5, \mathrm{R}_{c}$ tends to grow to infinity at the limit $\delta=-0.186$. This suggests that there is an asymptote where the critical $\mathbf{R}_{c}$ tends to infinity. This behavior corresponds to zone III in the $\beta-\delta$ plane displayed in Fig. 6. Figure 3(c) displays an example of a basic state in zone III. We notice that the corresponding perturbation (see Fig. 5) grows only at the outer part of the cylinder, where the basic temperature field locally corresponds to a linear flow, similar to that of homogeneous heating, although its isotherms are not strictly horizontal lines but are a bit bent. The part where the perturbation decays corresponds to a basic return flow, with rather vertical and slightly inverted isotherms. This feature suggests that the stationary localized structure is growing on a domain on which the vertical temperature gradient is negative and larger than a given critical value as occurs in the homogeneous heating case. This idea might be also useful for understanding the patterns in zone I, which seem to grow localized near the origin. A close look at the isotherms in Fig. 3(a) shows that in this case the return flow with inverted vertical temperature gradients is at the outer part of the cylinder while close to the origin the basic flow locally corresponds to a linear flow. Again the localized structure might be growing on a domain on which the vertical temperature gradient is negative and larger than a given critical value. This conjecture is difficult to test by formulating an equivalent problem in a smaller domain, as boundary conditions for these local flows are not easy to describe.

Finally, for $\delta<-0.186$ and $\beta=5$ no instability has been found in zone IV. This means that in this case there exist external parameter sets at which the axisymmetric basic state is very robust and does not bifurcate to $3 \mathrm{D}$ structures. This

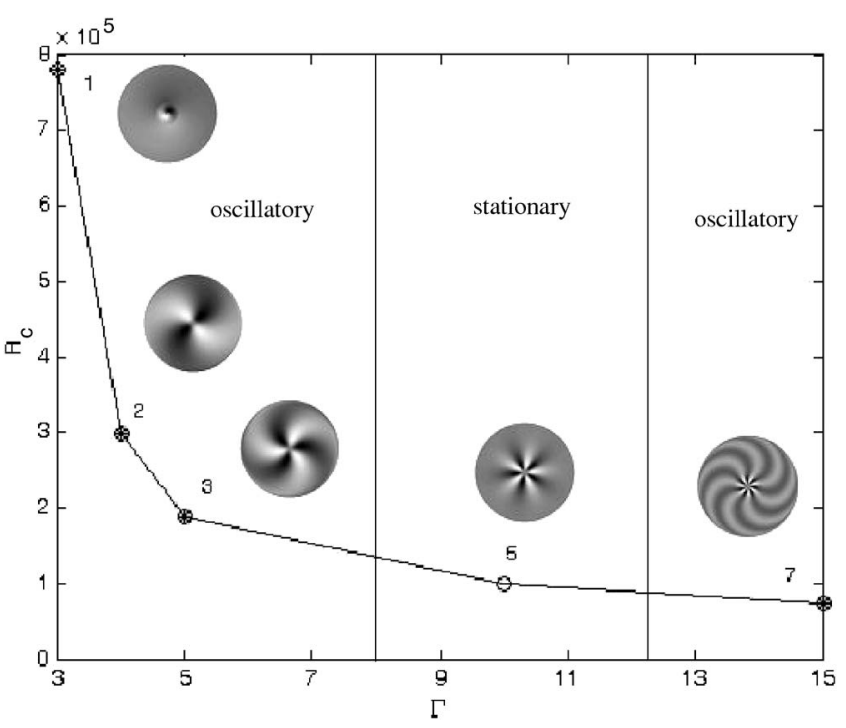

FIG. 9. Critical R, wave number $k$ values and growing modes as a function of $\Gamma$ for $\beta=0.5, \operatorname{Pr}=0.4, \mathrm{~B}=0.05$, and $\delta=1$.

regime is surrounded by curve $C_{3}$ (dashed line) in the $\beta-\delta$ diagram in Fig. 6. Figure 3(d) displays an example of a basic state in zone IV.

\section{Influence of the rest of the parameters}

Aspect ratio $\Gamma$. The results in the previous sections are based on a domain having a rather large aspect ratio $(\Gamma$ $=10$ ). At smaller aspect ratio the geometry of the domain has more influence on instabilities. Typically for uniform heating at smaller aspect ratio instability thresholds are larger than those obtained for larger aspect ratio, whereas critical wave numbers are smaller. Figure 9 shows how the instability thresholds and wave numbers change with $\Gamma$. It is observed that the standard rules just described for the case of uniform heating are maintained, although now oscillatory instabilities are possible. The same types of instabilities as those previously described are observed. We notice that in Fig. 9 for $\Gamma=3$ a one-armed spiral is the most unstable mode while the giant one-armed spirals, described before, were found to be less preferred than were the target patterns with $k=0$.

Pr number. Behaviors similar to those we have just described are also present for large and infinite Pr number. For instance, the giant one-armed oscillatory spiral appears as well for a similar range of values of the parameters $\beta$ and $\delta$ [see Fig. 8(c)], and with the same conditions, the oscillatory mode $k=1$ is just below the instability threshold but very close to it, at the same parameter values.

Biot number. In our study we have considered the Biot number to be $B=0.05$ except in Fig. 8(c), which takes the value $B=0.2$. The influence of the Biot number is important, as it fixes the heat exchange at the surface, and therefore the thermal state of the basic flow depends on it in the same way it depends on $\beta$ or $\delta$. A thorough study on how the Biot number affects all the above discussion is beyond the scope of this work, however, studies such as those in Refs. 14 and 15 describe such an influence in flows for which both buoyant and thermocapillary effects are present. In these refer- 
ences the fluid is in an annular container and the imposed temperature gradient at the bottom is constant. Typically one expects that at larger Biot number the critical temperature threshold decreases. ${ }^{14}$ The type of instability can be changed from stationary to oscillatory just by modifying the Biot number, and this allows the presence of numerous codimension-2 bifurcations points ${ }^{15}$ although these effects of the Biot number depend on the thermal state of the basic flow and may not be present for every choice of the external physical parameters. ${ }^{14}$ Our physical setup corresponds to buoyant flows, however, results on the effect of the Biot number could be similar to those including thermocapillary effects. For instance, in Ref. 31 it is found that instabilities and patterns of purely thermocapillary or purely buoyant flows are closely related.

\section{Discussion and conclusions}

We have studied how stationary and axisymmetric basic states bifurcate to different 3D structures depending on the shape factor $\beta$ and temperature ratio $\delta=\Delta T_{h} / \Delta T_{v}$ appearing on the temperature boundary condition. The Biot number is not varied in this work, however, as addressed in Refs. 14 and 15 it fixes the heat exchange at the surface and therefore affects the thermal state of the basic flow and the type of instabilities. The influence of horizontal and vertical temperature differences on instabilities has been addressed in numerous works. ${ }^{10,14-16}$ In our results a new heat parameter is introduced, which is related to the shape of the heat applied. We have found that at each $\beta$, different $\delta$ values define zones with qualitatively different instabilities. Typically, at small $\delta$ (and broad enough heating, i.e., large $\beta$ ) the basic state bifurcates to target and giant spiral wave patterns. The appearance of these structures is quite similar to those reported in experiments such as in Refs. 2-5 for the uniform heating case. The reason for this similarity could be the presence of a mean flow in those experiments similar to our basic state, which might be caused by a nonstrictly uniform heat source. In fact, the physical setup described in Sec. IV B 1, developing these instabilities, presents a spatial variation with a small gradient in the radial direction. We find that even for infinite Prandtl number spiral patterns are present. Larger negative $\delta$ values (i.e, an inhomogeneous heating source at the bottom which is colder at the center) lead to very localized states at the outer part of the domain. Localized structures in the center of the cell are found at small $\beta$ values and positive $\delta$ values (i.e., an inhomogeneous heating source at the bottom which is hotter at the center). A decreasing aspect ratio increases the instability threshold and decreases the critical wave number as happens in the uniform case.

\section{ACKNOWLEDGMENTS}

This work was partially supported by the Research Grant MCYT (Spanish Government) Nos. BFM2003-02832, MTM2004-00797, MTM2006-14843-C02-01, CCYT (JC de Castilla-La Mancha) Nos. PAC-05-005-01/02, SIMUMAT S-0505-ESP-0158 (Comunidad de Madrid), University of Castilla-La Mancha, and CSIC (Nos. PI-200650I224 and
PIF06-059). A.M.M. acknowledges MCYT (Spanish Government) for a Ramón y Cajal Research Fellowship and CESGA for computing support. The authors are grateful to D. Fox, E. Bodenschatz, and W. Pesch for useful discussions and comments.

${ }^{1} \mathrm{H}$. Bénard, "Les tourbillons cellulaires dans une nappe liquide," Rev. Gen. Sci. Pures Appl. 11, 1261-1271 (1900).

${ }^{2}$ E. Bodenschatz, J. R. de Bruyn, G. Ahlers, and D. S. Cannell, "Transitions between patterns in thermal convection," Phys. Rev. Lett. 67, 3078-3081 (1991).

${ }^{3}$ S. W. Morris, E. Bodenschatz, D. S. Cannell, and G. Ahlers, "Spiral defect chaos in large aspect ratio Rayleigh-Bénard convection," Phys. Rev. Lett. 71, 2026-2029 (1993).

${ }^{4}$ B. B. Plapp, D. A. Egolf, E. Bodenschatz, and W. Pesch, "Dynamics and selection of giant spirals in Rayleigh-Benard convection," Phys. Rev. Lett. 81, 5334-5337 (1998)

${ }^{5} \mathrm{M}$. Assenheimer, and V. Steinberg, "Transition between spiral and target states in Rayleigh-Bénard Convection," Nature 367, 345-347 (1994).

${ }^{6}$ M. Bestehorn, M. Fantz, R. Friedrich, H. Haken, and C. Perez-Garcia, "Spiral patterns in thermal convection," Z. Phys. B: Condens. Matter 88, 93-94 (1992).

${ }^{7} \mathrm{X}-\mathrm{J}$. Li, H-W. Xi, and J. D. Gunton, "Dynamical properties of multiarmed global spirals in Rayleigh-Bénard convection,” Phys. Rev. E 54, R3105R3108 (1996).

${ }^{8}$ W. Pesch, "Complex spatiotemporal convection patterns," Chaos 6, 348 357 (1996).

${ }^{9}$ W. Decker, W. Pesch, and A. Weber, "Spiral defect chaos in RayleighBénard convection," Phys. Rev. Lett. 73, 648-651 (1994).

${ }^{10}$ A. M. Mancho and H. Herrero, "Instabilities in a laterally heated liquid layer," Phys. Fluids 12, 1044-1051 (2000).

${ }^{11}$ A. M. Mancho, H. Herrero, and J. Burguete, "Primary instabilities in convective cells due to non-uniform heating," Phys. Rev. E 56, 2916-2923 (1997)

${ }^{12} \mathrm{H}$. Herrero and A. M. Mancho, "Influence of aspect ratio in convection due to non-uniform heating," Phys. Rev. E 57, 7336-7339 (1998).

${ }^{13}$ R. J. Riley and G. P. Neitzel, "Instability of thermocapillary-buoyancy convection in shallow layers. Part 1 . Characterization of steady and oscillatory instabilities," J. Fluid Mech. 359, 143-164 (1998).

${ }^{14}$ S. Hoyas, H. Herrero, and A. M. Mancho, "Thermal convection in a cylindrical annulus heated laterally," J. Phys. A 35, 4067-4083 (2002).

${ }^{15} \mathrm{~S}$. Hoyas, H. Herrero, and A. M. Mancho, "Bifurcation diversity of dynamic thermocapillary liquid layers," Phys. Rev. E 66, 057301 (2002).

${ }^{16}$ S. Hoyas, A. M. Mancho, H. Herrero, N. Garnier, and A. Chiffaudel, "Bénard-Marangoni convection in a differentially heated cylindrical cavity," Phys. Fluids 17, 054104 (2005).

${ }^{17}$ M. A. Pelacho and J. Burguete, "Temperature oscillations of hydrothermal waves in thermocapillary-buoyancy convection," Phys. Rev. E 59, 835840 (1999).

${ }^{18} \mathrm{H}$. Herrero and A. M. Mancho, "On pressure boundary conditions for thermoconvective problems," Int. J. Numer. Methods Fluids 39, 391-402 (2002).

${ }^{19}$ A. B. Ezersky, A. Garcimartín, J. Burguete, H. L. Mancini, and C. PérezGarcía, "Hydrothermal waves in Marangoni convection in a cylindrical container," Phys. Rev. E 47, 1126-1131 (1993).

${ }^{20} \mathrm{C}$. Bernardi and Y. Maday, Approximations Spectrales de Problèmes aux Limites Elliptiques (Springer-Verlag, Berlin, 1991).

${ }^{21}$ C. Canuto, M. Y. Hussaine, A. Quarteroni, and T. A. Zang, Spectral Methods in Fluid Dynamics (Springer, Berlin, 1988).

${ }^{22}$ H. Herrero, S. Hoyas, A. Donoso, A. M. Mancho, J. M. Chacn, R. F. Portugues, and B. Yeste, "Chebyshev collocation for a convective problem in primitive variables formulation,” J. Sci. Comput. 18, 315-328 (2003).

${ }^{23}$ M. C. Navarro, H. Herrero, A. M. Mancho, and A. Wathen, "Efficient solution of a generalized eigenvalue problem arising in a thermoconvective problem," Commun. Comput. Phys. (to be published).

${ }^{24}$ S. H. Davis, "On the principle of exchange of stabilities," Proc. R. Soc. London, Ser. A 310, 341-358 (1969).

${ }^{25} \mathrm{~N}$. Garnier and A. Chiffaudel, "Two dimensional hydrothermal waves in an extended cylindrical vessel," Eur. Phys. J. B 19, 87-95 (2001).

${ }^{26} \mathrm{~N}$. Garnier and C. Normand, "Effects of curvature on hydrothermal waves instability of radial thermocapillary flows," C. R. Acad. Sci., Ser. IV 2(8), 1227-1233 (2001)

${ }^{27}$ E. Favre, L. Blumenfeld, and F. Daviaud, "Instabilities of a liquid layer 
locally heated on its free surface," Phys. Fluids 9, 1473-1475 (1997).

${ }^{28}$ B. C. Sim, and A. Zebib, "Effect of free surface heat loss and rotation on transition to oscillatory thermocapillary convection," Phys. Fluids 14, 225-231 (2002).

${ }^{29}$ B. C. Sim, A. Zebib, and D. Schwabe, "Oscillatory thermocapillary convection in open cylindrical annuli. Part 2. Simulations," J. Fluid Mech. 491, 259-274 (2003).

${ }^{30}$ A. Pellew and R. V. Southwell, "On maintained convection in a fluid heated from below," Proc. R. Soc. London, Ser. A 176, 312-343 (1940).

${ }^{31}$ S. Hoyas, H. Herrero, and A. M. Mancho, "Thermocapillary and thermogravitatory waves in a convection problem," Theor. Comput. Fluid Dyn. 18, 309-321 (2002).

${ }^{32}$ M. K. Smith and S. H. Davis, "Instabilities of dynamic thermocapillary layers. 1. Convective instabilities," J. Fluid Mech. 132, 119-144 (1983).

${ }^{33}$ F. Daviaud and J. M. Vince, "Traveling waves in a fluid layer subjected to a horizontal temperature gradient," Phys. Rev. E 48, 4432-4436 (1993).

${ }^{34}$ J. Burguete, N. Mokolobwiez, F. Daviaud, N. Garnier, and A. Chiffaudel, "Buoyant-thermocapillary instabilities in extended layers subjected to a horizontal temperature gradient," Phys. Fluids 13, 2773-2787 (2001).

${ }^{35}$ S. Hoyas, "Estudio teórico y numérico de un problema de convección," Ph.D. thesis, Universidad Complutense de Madrid, 2003.

${ }^{36} \mathrm{~N}$. Garnier, "Ondes non-linéairesà une et deux dimensions dans une mince couche de fluide," Ph.D. thesis, University Paris 7 Denis Diderot, 2000.

${ }^{37}$ J. F. Mercier and C. Normand, "Buoyant-thermocapillary instabilities of differentially heated liquid layers," Phys. Fluids 8, 1433-1445 (1996). 